\title{
A Mathematical Characterization for Patterns of a Keller-Segel Model with a Cubic Source Term
}

\author{
Shengmao Fu ${ }^{1,2}$ and Ji Liu ${ }^{1}$ \\ ${ }^{1}$ College of Mathematics and Statistics, Northwest Normal University, Lanzhou 730070, China \\ ${ }^{2}$ School of Mathematics and Computing Science, Guilin University of Electronic Technology, Guilin 541004, China \\ Correspondence should be addressed to Shengmao Fu; fusm@nwnu.edu.cn
}

Received 4 October 2012; Revised 25 December 2012; Accepted 9 January 2013

Academic Editor: M. Lakshmanan

Copyright (c) 2013 S. Fu and J. Liu. This is an open access article distributed under the Creative Commons Attribution License, which permits unrestricted use, distribution, and reproduction in any medium, provided the original work is properly cited.

\begin{abstract}
This paper deals with a Neumann boundary value problem for a Keller-Segel model with a cubic source term in a $d$-dimensional box $(d=1,2,3)$, which describes the movement of cells in response to the presence of a chemical signal substance. It is proved that, given any general perturbation of magnitude $\delta$, its nonlinear evolution is dominated by the corresponding linear dynamics along a finite number of fixed fastest growing modes, over a time period of the order $\ln (1 / \delta)$. Each initial perturbation certainly can behave drastically differently from another, which gives rise to the richness of patterns. Our results provide a mathematical description for early pattern formation in the model.
\end{abstract}

\section{Introduction}

Keller and Segel in their pioneering work [1] proposed the following model

$$
\begin{gathered}
U_{t}=\nabla\left(D_{u} \nabla U-\chi U \nabla V\right), \\
V_{t}=D_{v} \nabla^{2} V+\alpha U-\beta V,
\end{gathered}
$$

where $U(x, t)$ is cell density, $V(x, t)$ is chemoattractant concentration, $D_{u}$ is the amoeboid motility, $\chi$ is the chemotactic sensitivity, $D_{v}$ is the diffusion rate of cyclic adenosine monophosphate (cAMP), $\alpha$ is the rate of cAMP secretion per unit density of amoebae, and $\beta$ is the rate of degradation of cAMP in environment. Keller and Segel wanted to model the chemotactic movement of the cellular slime mold Dictyostelium discoideum during its aggregation phase, where population growth does not occur. Therefore, they considered a population in the absence of "death" and "birth." For some main results on the Keller-Segel model, please see [2-4] and references therein.

Recently, Guo and Hwang in [5] investigated the nonlinear dynamics near an unstable constant equilibrium of the Keller-Segel model satisfying the Neumann boundary conditions for $U(x, t)$ and $V(x, t)$ on a $d$-dimensional box $\mathbb{T}^{d}=(0, \pi)^{d}(d=1,2,3)$; that is,

$$
\frac{\partial U}{\partial x_{i}}=\frac{\partial V}{\partial x_{i}}=0, \quad \text { at } x_{i}=0, \pi, \text { for } 1 \leq i \leq d .
$$

Let $[\bar{U}, \bar{V}]$ be the uniform constant solution of the KellerSegel model, and $u(x, t)=U(x, t)-\bar{U}, v(x, t)=V(x, t)-\bar{V}$. Then $[u, v]$ satisfies the equivalent Keller-Segel system below:

$$
\begin{gathered}
u_{t}=D_{u} \nabla^{2} u-\chi \bar{U} \nabla^{2} v-\chi \nabla(u \nabla v), \\
v_{t}=D_{v} \nabla^{2} v+\alpha u-\beta v
\end{gathered}
$$

Guo and Hwang proved that linear fastest growing modes determine unstable patterns for the above system. Their result can be interpreted as a rigorous mathematical characterization for early pattern formation in the Keller-Segel model.

In recent years, more and more attention has been given to the Keller-Segel model with the reaction terms, that is, the following chemotaxis-diffusion-growth model:

$$
\begin{gathered}
U_{t}=\nabla\left(D_{u} \nabla U-\chi U \nabla V\right)+f(U), \\
V_{t}=D_{v} \nabla^{2} V+\alpha U-\beta V .
\end{gathered}
$$


For $f(U)=r U(1-U / K)$, Painter and Hillen [6] demonstrated the capacity of the above model to self-organize into multiple cellular aggregations which, according to its position in parameter space, either form a stationary pattern or undergo a sustained spatiotemporal sequence of merging (two aggregations coalesce) and emerging (a new aggregation appears). This spatiotemporal patterning can be further subdivided into either a time-periodic or time-irregular fashion. Numerical explorations into the latter indicate a positive Lyapunov exponent (sensitive dependence to initial conditions) together with a rich bifurcation structure. In particular, they found stationary patterns that bifurcate onto a path of periodic patterns which, prior to the onset of spatiotemporal irregularity, undergo a "periodic-doubling" sequence. Based on these results and comparisons with other systems, they argued that the spatiotemporal irregularity observed here describes a form of spatiotemporal chaos.

For $f(U)=r U(1-U)$, Banerjee et al. [7] showed that the dynamics of the chemotaxis-diffusion-growth model may lead to steady states, to divergencies in a finite time, and to the formation of spatiotemporal irregular patterns. The latter, in particular, appears to be chaotic in part of the range of bounded solutions, as demonstrated by the analysis of wavelet power spectra. Steady states are achieved with sufficiently large values of the chemotactic coefficient $\chi$ and/or with growth rate $r$ below a critical value $r_{c}$. For $r>r_{c}$, the solutions of the differential equations of the model diverge in a finite time. They also reported on the pattern formation regime, for different value of $\chi, r$ and of the diffusion coefficient $D_{u}$. For the same $f(U)$, Kuto et al. [8] considered some qualitative behaviors of stationary solutions from global and local (bifurcation) viewpoints. They studied the asymptotic behavior of stationary solutions as the chemotactic intensity grows to infinity and construct local bifurcation branches of stripe and hexagonal stationary solutions in the special case when the habitat domain is a rectangle. For this case, the directions of the branches near the bifurcation points are also obtained. Finally, they exhibited several numerical results for the stationary and oscillating patterns.

In [9], Okuda and Osaki studied the chemotaxis-diffusion-growth model with $f=p U(1-U)+q U(1-U)(U-v)$ in a rectangular domain by applying the center manifold theory, where constant $v \in(0,1)$ and either $p>0, q=0$, or $p=0, q>0$. It is observed that the trivial solutions are destabilized due to the chemotaxis term. They obtained the normal form on the center manifold, and it is proved that the locally asymptotically stable hexagonal patterns exist.

Another extended formation of logistic source term is the cubic source term $f=U\left(b_{1}+b_{2} U-b_{3} U^{2}\right)$, where $b_{1} \geq 0$ is the intrinsic growth rate, the sign of $b_{2}$ is undetermined, $b_{3}>0$ is a positive constant, and $b_{2} U-b_{3} U^{2}$ is the density restriction term (see $[10,11]$ for more information and references). Recently, Cao and $\mathrm{Fu}$ in [11] studied global existence and convergence of solutions to a cross-diffusion cubic predatorprey system with stage structure for the prey. In this paper, we investigate dynamics of the chemotaxis-diffusion-growth model with the source term $f=\left(b_{1}+b_{2} U-b_{3} U^{2}\right) U$; that is,

$$
\begin{aligned}
U_{t}= & \nabla\left(D_{u} \nabla U-\chi U \nabla V\right) \\
& +\left(b_{1}+b_{2} U-b_{3} U^{2}\right) U, \\
V_{t}= & D_{v} \nabla^{2} V+\alpha U-\beta V,
\end{aligned}
$$

where $D_{u}, \chi, D_{v}, \alpha$, and $\beta$ are positive constants and $U(x, t)$, $V(x, t)$ satisfies the Neumann boundary conditions. We will prove that given any general perturbation of magnitude $\delta$, its nonlinear evolution is dominated by the corresponding linear dynamics along a fixed finite number of fastest growing modes, over a time period of $\ln (1 / \delta)$. Each initial perturbation certainly can behave drastically differently from another, which gives rise to the richness of patterns. Our results provide a mathematical description for early pattern formation in the model (5).

The organization of this paper is as follows: In Section 2, we prove that the positive constant equilibrium solution of (5) without chemotaxis is globally asymptotically stable if $b_{1}>0$. In Section 3, we investigate the growing modes of (5). In Section 4, we present and prove the Bootstrap lemma. In Section 5, given any general perturbation of magnitude $\delta$, we prove that its nonlinear evolution is dominated by the corresponding linear dynamics along a fixed finite number of fastest growing modes, over a time period of $\ln (1 / \delta)$.

\section{Stability of Positive Equilibrium Point of (5) without Chemotaxis}

The corresponding semilinear system of (5) without chemotaxis is as follows:

$$
\begin{gathered}
U_{t}=D_{u} \nabla^{2} U+\left(b_{1}+b_{2} U-b_{3} U^{2}\right) U, \quad x \in \mathbb{T}^{d}, t>0, \\
V_{t}=D_{v} \nabla^{2} V+\alpha U-\beta V, \quad x \in \mathbb{T}^{d}, t>0, \\
\frac{\partial U}{\partial x_{i}}=\frac{\partial V}{\partial x_{i}}=0, \quad x_{i}=0, \pi, \quad 1 \leq i \leq d, \\
U(x, 0)=U_{0}(x) \geq 0, \quad x \in \mathbb{T}^{d}(d=1,2,3) . \\
V(x, 0)=V_{0}(x) \geq 0, \quad
\end{gathered}
$$

Obviously, $[\bar{U}, \bar{V}] \equiv\left[\left(b_{2}+\sqrt{b_{2}^{2}+4 b_{1} b_{3}}\right) / 2 b_{3}, \alpha\left(b_{2}+\right.\right.$ $\left.\sqrt{b_{2}^{2}+4 b_{1} b_{3}}\right) / 2 \beta b_{3}$ ] is a positive equilibrium point of (6) if and only if either of the following two cases happens: (i) $b_{1}>$ $0, b_{2} \in \mathbb{R}$, (ii) $b_{1}=0, b_{2}>0$. In the following we will discuss the stability of $[\bar{U}, \bar{V}]$ in (6).

Let $W=[U, V], \bar{W}=[\bar{U}, \bar{V}]$, and $0=\mu_{1}<\mu_{2}<$ $\mu_{3}<\cdots$ be the eigenvalues of the operator $-\Delta$ on $\mathbb{T}^{d}(d=$ $1,2,3)$ with the homogeneous Neumann boundary condition, and let $E\left(\mu_{i}\right)$ be the eigenspace corresponding to $\mu_{i}$ in $L^{2}\left(\mathbb{T}^{d}\right)$. Let $X=\left[L^{2}\left(\mathbb{T}^{d}\right)\right]^{2},\left\{\phi_{i j}: j=1, \ldots, \operatorname{dim} E\left(\mu_{i}\right)\right\}$ be an orthonormal basis of $E\left(\mu_{i}\right)$, and $X_{i j}=\left\{\mathbf{c} \cdot \phi_{i j} \mid \mathbf{c} \in \mathbb{R}^{2}\right\}$. Then

$$
X=\oplus_{i=1}^{\infty} X_{i}, \quad X_{i}=\oplus_{j=1}^{\operatorname{dim}\left[E\left(\mu_{i}\right)\right]} X_{i j} .
$$


Let $\mathfrak{D}=\operatorname{diag}\left(D_{u}, D_{v}\right)$ and $\mathfrak{Q}=\mathfrak{D} \Delta+G_{W}(\bar{W})$, where

$$
G_{W}(\bar{W})=\left(\begin{array}{cc}
-3 b_{3} \bar{U}^{2}+2 b_{2} \bar{U}+b_{1} & 0 \\
\alpha & -\beta
\end{array}\right) .
$$

Then the linearization of (6) at $[\bar{U}, \bar{V}]$ is $W_{t}=\mathfrak{Q}(W-\bar{W})$.

For each $i \geq 1, X_{i}$ is invariant under the operator $\mathfrak{L}$, and $\lambda$ is an eigenvalue of $\mathcal{Q}$ on $X_{i}$ if and only if it is an eigenvalue of the matrix

$$
\begin{aligned}
-\mu_{i} \mathfrak{D} & +G_{W}(\bar{W}) \\
& =\left(\begin{array}{cc}
-\mu_{i} D_{u}-3 b_{3} \bar{U}^{2}+2 b_{2} \bar{U}+b_{1} & 0 \\
\alpha & -\mu_{i} D_{v}-\beta
\end{array}\right) .
\end{aligned}
$$

Notice that $\left(b_{2}+\sqrt{b_{2}^{2}+3 b_{1} b_{3}}\right) / 3 b_{3}$ is the positive root of $-3 b_{3} x^{2}+2 b_{2} x+b_{1}=0$ and

$$
\bar{U}>\frac{b_{2}+\sqrt{b_{2}^{2}+3 b_{1} b_{3}}}{3 b_{3}} .
$$

Thus, $-\mu_{i} \mathfrak{D}+G_{W}(\bar{W})$ has two negative eigenvalues $-\mu_{i} D_{u}-$ $3 b_{3} \bar{U}^{2}+2 b_{2} \bar{U}+b_{1}$ and $-\mu_{i} D_{v}-\beta$. It follows from [12, Theorem 5.1.1] that $[\bar{U}, \bar{V}]$ is locally asymptotically stable.

Let $[U, V]$ be a unique nonnegative global solution of (6). It is not hard to verify by the maximum principle that

$$
\begin{aligned}
& 0 \leq U \leq \max \left\{\bar{U},\left|U_{0}(x)\right|_{\infty}\right\}, \quad \forall t \geq 0 . \\
& 0 \leq V \leq \max \left\{\bar{V},\left|V_{0}(x)\right|_{\infty}\right\},
\end{aligned}
$$

Moreover, if $U_{0}, V_{0} \geq(\neq \equiv) 0$, then $U(x, t)>0, V(x, t)>0$, for all $t>0$.

According to the main result in [13], we have

$$
\begin{aligned}
& \|U(\cdot, t)\|_{C^{2, \alpha}\left(\overline{\mathbb{T}}^{d}\right)} \leq C, \quad \forall t \geq 1 . \\
& \|V(\cdot, t)\|_{C^{2, \alpha}\left(\overline{\mathbb{T}}^{d}\right)} \leq C, \quad \forall t
\end{aligned}
$$

(i) If $b_{1}>0, b_{2} \in \mathbb{R}$, then $b_{3} \bar{U}-b_{2}>0$. We define the Lyapunov function

$$
E(t)=\int_{\mathbb{T}^{d}}\left[p\left(U-\bar{U}-\bar{U} \ln \frac{U}{\bar{U}}\right)+(V-\bar{V})^{2}\right] d x,
$$

where $p=2 \alpha^{2} /\left(\beta\left(b_{3} \bar{U}-b_{2}\right)\right)$.
Calculating the derivative of $E(t)$ along positive solution of (6) by integration by parts and the Cauchy inequality, we have

$$
\begin{aligned}
& \frac{d E(u, v)}{d t} \\
& \leq-\int_{\mathbb{J}^{d}}\left\{\frac{p D_{u} \bar{U}}{U^{2}}|\nabla U|^{2}+2 D_{v}|\nabla V|^{2}\right\} d x \\
& \quad-\int_{\mathbb{T}^{d}}\left\{p\left(b_{3} U+b_{3} \bar{U}-b_{2}\right)(U-\bar{U})^{2}\right. \\
& \left.-2 \alpha(U-\bar{U})(V-\bar{V})+2 \beta(V-\bar{V})^{2}\right\} d x \\
& \leq-\int_{\mathbb{T}^{d}}\left\{p\left(b_{3} \bar{U}-b_{2}\right)(U-\bar{U})^{2}\right. \\
& \left.\quad-2 \alpha(U-\bar{U})(V-\bar{V})+2 \beta(V-\bar{V})^{2}\right\} d x \\
& \leq-\frac{\alpha^{2}}{\beta} \int_{\mathbb{T}^{d}}(U-\bar{U})^{2} d x-\beta \int_{\mathbb{T}^{d}}(V-\bar{V})^{2} d x .
\end{aligned}
$$

(ii) If $b_{1}=0, b_{2}>0$, then $b_{2}=b_{3} \bar{U}$. We define the Lyapunov function

$$
E(t)=\int_{\mathbb{T}^{d}}\left[\frac{q}{U}(U-\bar{U})^{2}(V-\bar{V})^{2}\right] d x,
$$

where $q=2 \alpha^{2} / \beta b_{3} \bar{U}$.

Calculating in the same way as (14), we have

$$
\begin{aligned}
\frac{d E(u, v)}{d t} \leq & -\int_{\mathbb{T}^{d}} 2 \frac{q D_{u} \bar{U}^{2}}{u^{3}}|\nabla U|^{2}+D_{v}|\nabla V|^{2} d x \\
& -\int_{\mathbb{T}^{d}} q b_{3}(U+\bar{U})(U-\bar{U})^{2} \\
& \quad-2 \alpha(U-\bar{U})(V-\bar{V})+2 \beta(V-\bar{V})^{2} d x \\
\leq & -\int_{\mathbb{T}^{d}} q b_{3} \bar{U}(U-\bar{U})^{2}-2 \alpha(U-\bar{U})(V-\bar{V}) \\
& \quad+2 \beta(V-\bar{V})^{2} d x \\
\leq & -\frac{\alpha^{2}}{\beta} \int_{\mathbb{T}^{d}}(U-\bar{U})^{2} d x-\beta \int_{\mathbb{T}^{d}}(V-\bar{V})^{2} d x .
\end{aligned}
$$

Combining (12)-(16) and Lemma 3.2 in [11], we conclude that

$$
\begin{aligned}
& \lim _{t \rightarrow \infty}\|U(\cdot, t)-\bar{U}\|_{L^{2}\left(\mathbb{T}^{d}\right)}=0, \\
& \lim _{t \rightarrow \infty}\|V(\cdot, t)-\bar{V}\|_{L^{2}\left(\mathbb{T}^{d}\right)}=0 .
\end{aligned}
$$

The global asymptotic stability of $[\bar{U}, \bar{V}]$ follows from (17) and the local stability of $[\bar{U}, \bar{V}]$. 
Theorem 1. The positive equilibrium point $[\bar{U}, \bar{V}]$ of (6) is locally asymptotically stable. If either $b_{1}>0, b_{2} \in \mathbb{R}$ or $b_{1}=0$, $b_{2}>0$ holds, then $[\bar{U}, \bar{V}]$ is globally asymptotically stable.

\section{Growing Modes in the System (5)}

Let $u(\mathbf{x}, t)=U(\mathbf{x}, t)-\bar{U}, v(\mathbf{x}, t)=V(\mathbf{x}, t)-\bar{V}$. Then

$$
\begin{gathered}
u_{t}=D_{u} \nabla^{2} u-\chi \bar{U} \nabla^{2} v-\left(3 b_{3}^{2} \bar{U}^{2}-2 b_{2} \bar{U}-b_{1}\right) u \\
-\chi \nabla(u \nabla v)+\left(b_{2}-3 b_{3} \bar{U}\right) u^{2}-b_{3} u^{3}, \\
v_{t}=D_{v} \nabla^{2} v+\alpha u-\beta v .
\end{gathered}
$$

The corresponding linearized system takes the form

$$
\begin{gathered}
u_{t}=D_{u} \nabla^{2} u-\chi \bar{U} \nabla^{2} v-\left(3 b_{3}^{2} \bar{U}^{2}-2 b_{2} \bar{U}-b_{1}\right) u, \\
v_{t}=D_{v} \nabla^{2} v+\alpha u-\beta v .
\end{gathered}
$$

Let $\mathbf{w}(\mathbf{x}, t) \equiv[u(\mathbf{x}, t), v(\mathbf{x}, t)], \mathbf{q}=\left(q_{1}, \ldots, q_{d}\right) \in \Omega=\mathbb{N}^{d}$, and $e_{\mathbf{q}}(\mathbf{x})=\prod_{i=1}^{d} \cos \left(q_{i} x_{i}\right)$. Then $\left\{e_{\mathbf{q}}(\mathbf{x})\right\}_{\mathbf{q} \in \Omega}$ forms a basis of the space of functions in $\mathbb{T}^{d}$ that satisfy Neumann boundary conditions. We look for a normal mode to the linearized system (19) of the following form

$$
\mathbf{w}(\mathbf{x}, t)=\mathbf{r}_{\mathbf{q}} \exp \left(\lambda_{\mathbf{q}} t\right) e_{\mathbf{q}}(\mathbf{x}),
$$

where $\mathbf{r}_{\mathbf{q}}$ is a vector depending on $\mathbf{q}$. Plugging (20) into (19) we have the following dispersion formula for $\lambda_{\mathbf{q}}$

$$
\begin{gathered}
\lambda_{\mathbf{q}}^{2}+\left\{q^{2}\left(D_{u}+D_{v}\right)+\beta+3 b_{3} \bar{U}^{2}-2 b_{2} \bar{U}-b_{1}\right\} \lambda_{\mathbf{q}} \\
+q^{2}\left\{D_{u} D_{v} q^{2}+\beta D_{u}+\left(3 b_{3} \bar{U}^{2}-2 b_{2} \bar{U}-b_{1}\right) D_{v}\right. \\
-\alpha \chi \bar{U}\}+\left(3 b_{3} \bar{U}^{2}-2 b_{2} \bar{U}-b_{1}\right) \beta=0 .
\end{gathered}
$$

Thus we deduce the following well-known aggregation (i.e., linear instability) criterion by requiring there exists a $\mathbf{q}$ such that

$$
\begin{gathered}
q^{2}\left\{D_{u} D_{v} q^{2}+\beta D_{u}+\left(3 b_{3} \bar{U}^{2}-2 b_{2} \bar{U}-b_{1}\right) D_{v}-\alpha \chi \bar{U}\right\} \\
+\left(3 b_{3} \bar{U}^{2}-2 b_{2} \bar{U}-b_{1}\right) \beta<0
\end{gathered}
$$

to ensure that (21) has at least one positive root $\lambda_{\mathbf{q}}$. This implies that for $\mathbf{q}$,

$$
\begin{aligned}
& \left\{q^{2}\left(D_{u}+D_{v}\right)+\beta+3 b_{3} \bar{U}^{2}-2 b_{2} \bar{U}-b_{1}\right\}^{2} \\
& -4\left\{q ^ { 2 } \left\{D_{u} D_{v} q^{2}+\beta D_{u}+\left(3 b_{3} \bar{U}^{2}-2 b_{2} \bar{U}-b_{1}\right) D_{v}\right.\right. \\
& \left.-\alpha \chi \bar{U}\}+\left(3 b_{3} \bar{U}^{2}-2 b_{2} \bar{U}-b_{1}\right) \beta\right\} \\
& =\left\{q^{2}\left(D_{u}-D_{v}\right)-\left(\beta-3 b_{3} \bar{U}^{2}+2 b_{2} \bar{U}+b_{1}\right)\right\}^{2} \\
& +4 \alpha \chi \bar{U} q^{2} \geq 0 .
\end{aligned}
$$

If $\mathbf{q}=\mathbf{0}$, then (21) has two negative roots $-\beta$ and $-3 b_{3} \bar{U}^{2}+$ $2 b_{2} \bar{U}+b_{1}$. Therefore, the positive equilibrium point of (18) is locally asymptotically stable.

Now we investigate nonlinear dynamics near the unstable constant equilibrium solution of (18) in the case $\mathbf{q} \neq \mathbf{0}$. If $\mathbf{q} \neq \mathbf{0}$, the right side of (23) is positive. Therefore, there exist two distinct real roots $\lambda_{\mathbf{q}}^{ \pm}$for all $\mathbf{q} \neq \mathbf{0}$ to the quadratic equation (21). We denote the corresponding (linearly independent) eigenvectors by $\mathbf{r}_{-}(\mathbf{q})$ and $\mathbf{r}_{+}(\mathbf{q})$, such that

$$
\mathbf{r}_{ \pm}(\mathbf{q})=\left[\frac{\lambda_{\mathbf{q}}^{ \pm}+D_{v} q^{2}+\beta}{\alpha}, 1\right] .
$$

Clearly, for $q$ large

$$
\begin{aligned}
q^{2}\left\{D_{u} D_{v} q^{2}+\beta D_{u}+\left(3 b_{3} \bar{U}^{2}-2 b_{2} \bar{U}-b_{1}\right) D_{v}-\alpha \chi \bar{U}\right\} \\
+\left(3 b_{3} \bar{U}^{2}-2 b_{2} \bar{U}-b_{1}\right) \beta>0 .
\end{aligned}
$$

Hence, there are only finitely many $\mathbf{q}$ such that $\lambda_{\mathbf{q}}^{+}>0$. We denote the largest eigenvalue by $\lambda_{\max }>0$ and define $\Omega_{\max } \equiv\left\{\mathbf{q} \in \Omega \mid \lambda_{\mathbf{q}}^{+}=\lambda_{\max }\right\}$. It is easy to see that there is one $q^{2}$ (possibly two) having $\lambda^{+}\left(q^{2}\right)=\lambda_{\max }$ if we regard $\lambda_{\mathbf{q}}^{+}$as a function of $q^{2}$. We also denote $v>0$ to be the gap between the $\lambda_{\max }$ and the rest. Given any initial perturbation $\mathbf{w}(\mathbf{x}, 0)$, we can expand it as

$$
\begin{aligned}
\mathbf{w}(\mathbf{x}, 0) & =\sum_{\mathbf{q} \in \Omega} \mathbf{w}_{\mathbf{q}} e_{\mathbf{q}}(\mathbf{x}) \\
& =\sum_{\mathbf{q} \in \Omega}\left\{w_{\mathbf{q}}^{-} \mathbf{r}_{-}(\mathbf{q})+w_{\mathbf{q}}^{+} \mathbf{r}_{+}(\mathbf{q})\right\} e_{\mathbf{q}}(\mathbf{x}),
\end{aligned}
$$

so that

$$
\mathbf{w}_{\mathbf{q}}=w_{\mathbf{q}}^{-} \mathbf{r}_{-}(\mathbf{q})+w_{\mathbf{q}}^{+} \mathbf{r}_{+}(\mathbf{q}) .
$$

The unique solution $\mathbf{w}(\mathbf{x}, t)=[u(\mathbf{x}, t), v(\mathbf{x}, t)]$ to (19) is given by

$$
\begin{aligned}
& \mathbf{w}(\mathbf{x}, t) \\
&=\sum_{\mathbf{q} \in \Omega}\left\{w_{\mathbf{q}}^{-} \mathbf{r}_{-}(\mathbf{q}) \exp \left(\lambda_{\mathbf{q}}^{-} t\right)\right. \\
&\left.\quad+w_{\mathbf{q}}^{+} \mathbf{r}_{+}(\mathbf{q}) \exp \left(\lambda_{\mathbf{q}}^{+} t\right)\right\} e_{\mathbf{q}}(\mathbf{x}) \equiv e^{\Omega(t} \mathbf{w}(x, 0) .
\end{aligned}
$$

For any $\mathbf{g}(\cdot, t) \in\left[L^{2}\left(\mathbb{T}^{d}\right)\right]^{2}$, we denote $\|\mathbf{g}(\cdot, t)\| \equiv\|\mathbf{g}(\cdot, t)\|_{L^{2} \text {. }}$. Our main result of this section is the following lemma.

Lemma 2. Suppose that the instability criterion (22) holds. Let $\mathbf{w}(\mathbf{x}, t) \equiv e^{\mathfrak{R} t} \mathbf{w}(\mathbf{x}, 0)$ be a solution to the linearized system (19) with initial condition $\mathbf{w}(\mathbf{x}, 0)$. Then there exists a constant $C_{1} \geq$ 1 depending on $D_{u}, \chi, \bar{U}, b_{1}, b_{2}, b_{3}, D_{v}, \alpha$, and $\beta$, such that

$$
\|\mathbf{w}(\cdot, t)\| \leq C_{1} \exp \left(\lambda_{\max } t\right)\|\mathbf{w}(\cdot, 0)\|, \quad \forall t \geq 0 .
$$


Proof. We first consider the case for $t \geq 1$. By analyzing (21), for $q$ large, we have

$$
\lim _{q \rightarrow \infty} \frac{\lambda_{\mathbf{q}}^{ \pm}}{q^{2}}=-D_{u},-D_{v},
$$

respectively. From the quadratic formula of (21), we can see that

$$
\frac{\lambda_{\mathbf{q}}^{+}-\lambda_{\mathbf{q}}^{-}}{q^{2}} \geq \frac{2 \sqrt{\alpha \chi \bar{U}}}{q} .
$$

It follows from (27) that

$$
\left|w_{\mathbf{q}}^{ \pm}\right| \leq \frac{\left|\mathbf{r}_{ \pm}(\mathbf{q})\right| \times\left|\mathbf{w}_{\mathbf{q}}\right|}{\left|\operatorname{det}\left[\mathbf{r}_{-}(\mathbf{q}), \mathbf{r}_{+}(\mathbf{q})\right]\right|} .
$$

Later on we will always denote universal constants by $E_{i}(i=$ $1,2, \ldots$ ). Note that $\mathbf{q} \in \mathbb{N}^{d}$ and $\mathbf{q} \neq \mathbf{0}$. From (24) and (30), for all $q>0$, there exists a positive constant $E_{1}$ and $E_{2}$, such that $\left|\lambda_{\mathbf{q}}^{ \pm} / \alpha q^{2}\right| \leq E_{1}$, and

$$
\left|\mathbf{r}_{ \pm}(\mathbf{q})\right| \leq E_{2} q^{2} \text {. }
$$

By (24), (31), (32), and (33), we deduce that

$$
\left|w_{\mathbf{q}}^{ \pm}\right| \leq E_{3} q\left|\mathbf{w}_{\mathbf{q}}\right| \text {. }
$$

Thus, it is clear from (33) and (34) that

$$
\left|w_{\mathbf{q}}^{ \pm} \mathbf{r}_{ \pm}(\mathbf{q}) \exp \left(\lambda_{\mathbf{q}}^{ \pm} t\right)\right| \leq E_{2} E_{3} q^{3}\left|\mathbf{w}_{\mathbf{q}}\right| \exp \left(\lambda_{\mathbf{q}}^{ \pm} t\right) .
$$

For $t \geq 1$, it is not hard to verify that there exists a constant $E_{4}>0$, such that

$$
q^{3} \exp \left(\lambda_{\mathbf{q}}^{ \pm} t\right) \leq E_{4}
$$

It follows from (35) and (36) that

$$
\left|w_{\mathbf{q}}^{ \pm} \mathbf{r}_{ \pm}(\mathbf{q}) \exp \left(\lambda_{\mathbf{q}}^{ \pm} t\right)\right| \leq E_{5}\left|\mathbf{w}_{\mathbf{q}}\right| \text {. }
$$

Denote by $\langle\cdot, \cdot\rangle$ and $(\cdot, \cdot)$ the inner product of $\left[L^{2}\left(\mathbb{T}^{d}\right)\right]^{2}$ and the scaler product of $\mathbb{R}^{2}$, respectively. A simple computation shows that

$$
\|\mathbf{w}(\mathbf{x}, 0)\|^{2}=\left(\frac{\pi}{2}\right)^{d} \sum_{\mathbf{q} \in \Omega}\left|\mathbf{w}_{\mathbf{q}}\right|^{2} .
$$

From (28), (37) and (38), we have

$$
\|\mathbf{w}(\mathbf{x}, t)\| \leq 2 E_{5} \exp \left(\lambda_{\max } t\right)\|\mathbf{w}(\mathbf{x}, 0)\|, \quad \text { for } t \geq 1 .
$$

On the other hand, for $t<1$, it is sufficient to derive the standard energy estimate in $L^{2}$. By (19), we have

$$
\begin{aligned}
& \frac{1}{2} \frac{d}{d t} \int_{\mathbb{T}^{d}}\left\{|u|^{2}+A|v|^{2}\right\} \mathbf{d} \mathbf{x} \\
& \quad+\int_{\mathbb{T}^{d}}\left\{D_{u}|\nabla u|^{2}+A D_{v}|\nabla v|^{2}-\chi \bar{U} \nabla u \nabla v\right\} \mathbf{d} \mathbf{x} \\
& \quad+\left(3 b_{3} \bar{U}^{2}-2 b_{2} \bar{U}-b_{1}\right) \int_{\mathbb{T}^{d}} u^{2} \mathbf{d} \mathbf{x} \\
& \quad+A \beta \int_{\mathbb{T}^{d}} v^{2} \mathbf{d} \mathbf{x}=A \alpha \int_{\mathbb{T}^{d}} u v \mathbf{d} \mathbf{x} .
\end{aligned}
$$

Let

$$
A=\frac{(\chi \bar{U})^{2}}{D_{u} D_{v}} .
$$

Then the integrand of the second term on the left side of (40) satisfies

$$
\begin{aligned}
D_{u}|\nabla u|^{2}+A D_{v}|\nabla v|^{2}-\chi \bar{U} \nabla u \nabla v \\
\geq \frac{D_{u}}{2}|\nabla u|^{2}+\frac{(\chi \bar{U})^{2}}{2 D_{u}}|\nabla v|^{2} \geq 0 .
\end{aligned}
$$

By (40), Young's inequality, and $A \geq 1$, we deduce that

$$
\frac{1}{2} \frac{d}{d t} \int_{\mathbb{V}^{d}}\left\{|u|^{2}+A|v|^{2}\right\} \mathbf{d x} \leq \frac{A \alpha}{2} \int_{\mathbb{T}^{d}}\left\{|u|^{2}+A|v|^{2}\right\} \mathbf{d x} .
$$

Using Grownwall's inequality and noticing $A \geq 1$ and $t<1$, we can obtain

$$
\|\mathbf{w}(\mathbf{x}, t)\| \leq(A \exp (A \alpha))^{1 / 2} \exp \left(\lambda_{\max } t\right)\|\mathbf{w}(\mathbf{x}, 0)\| .
$$

If $0<A<1$, by (43), $t<1$, and Grownwall inequality, we have

$$
\|\mathbf{w}(\mathbf{x}, t)\| \leq\left(\frac{\exp \alpha}{A}\right)^{1 / 2} \exp \left(\lambda_{\max } t\right)\|\mathbf{w}(\mathbf{x}, 0)\| .
$$

Let $C_{1}=\max \left\{2 E_{5},(A \exp (A \alpha))^{1 / 2}\right\} \geq 1$ if $0<A<1$. Then $\|\mathbf{w}(\mathbf{x}, t)\| \leq C_{1} \exp \left(\lambda_{\max } t\right)\|\mathbf{w}(\mathbf{x}, 0)\|$ if $A>1$ and $C_{1}=$ $\max \left\{2 E_{5},(\exp \alpha / A)^{1 / 2}\right\} \geq 1$.

\section{Bootstrap Lemma}

By a standard PDE theory [14], we can establish the existence of local solutions for (18).

Lemma 3 (local existence). For $s \geq 1(d=1)$ and $s \geq 2(d=$ $2,3)$, there exists $T_{0}>0$, such that $(18)$ with $u(\cdot, 0), v(\cdot, 0) \in H^{s}$ has a unique solution $\mathbf{w}(\cdot, t)$ on $\left(0, T_{0}\right)$ which satisfies

$$
\|\mathbf{w}(t)\|_{H^{s}} \leq C\|\mathbf{w}(0)\|_{H^{s}}, \quad 0<t<T_{0}
$$

where $C$ is a positive constant depending on $D_{u}, \chi, \bar{U}, b_{1}, b_{2}, b_{3}$, $D_{v}, \alpha$, and $\beta$.

It is not hard to verify the following result.

Lemma 4. Let $\mathbf{w}(\mathbf{x}, t)$ be a solution of (18). Then the even extension of $\mathbf{w}(\mathbf{x}, t)$ on $2 \mathbb{T}^{d}=(-\pi, \pi)^{d}(d=1,2,3)$ is also the solution of (18) which satisfies homogeneous Neumann boundary conditions and periodical boundary conditions on $2 \mathbb{T}^{d}=(-\pi, \pi)^{d}(d=1,2,3)$ 
Lemma 5. Let $[u(\mathbf{x}, t), v(\mathbf{x}, t)]$ be a solution of (18). Then

$$
\begin{aligned}
& \frac{1}{2} \frac{d}{d t} \sum_{|\alpha|=2} \int_{\mathbb{T}^{d}}\left\{\left|D^{\alpha} u\right|^{2}+\frac{(\chi \bar{U})^{2}}{D_{u} D_{v}}\left|D^{\alpha} v\right|^{2}\right\} \mathbf{d} \mathbf{x} \\
& +\sum_{|\alpha|=2} \int_{\mathbb{T}^{d}}\left\{\frac{D_{u}}{4}\left|\nabla D^{\alpha} u\right|^{2}+\frac{(\chi \bar{U})^{2}}{2 D_{u}}\left|\nabla D^{\alpha} v\right|^{2}\right\} \mathbf{d} \mathbf{x} \\
& +\frac{A \beta}{2} \sum_{|\alpha|=2} \int_{\mathbb{T}^{d}}\left|D^{\alpha} v\right|^{2} \mathbf{d x}+\frac{3 b_{3} \bar{U}^{2}-2 b_{2} \bar{U}-b_{1}}{2} \\
& \quad \times \sum_{|\alpha|=2} \int_{\mathbb{T}^{d}}\left|D^{\alpha} u\right|^{2} \mathbf{d} \mathbf{x} \leq C_{0}\left(\frac{\chi}{2}+\left|b_{2}-3 b_{3} \bar{U}\right|+\frac{3}{2} b_{3}\right) \\
& \quad \times\left(\|\mathbf{w}\|_{H^{2}}+\|\mathbf{w}\|_{H^{2}}^{2}\right)\left\|\nabla^{3} \mathbf{w}\right\|^{2}+C_{2}\|u\|^{2},
\end{aligned}
$$

where $C_{2}=E_{15} \chi^{12} \bar{U}^{12} \alpha^{12} / 2 D_{u}^{8} D_{v}^{6} \beta^{6}\left(3 b_{3} \bar{U}^{2}-2 b_{2} \bar{U}-b_{1}\right)^{3}$.

Proof. It is known by Lemma 4 that

$$
\begin{gathered}
\tilde{u}_{t}=D_{u} \nabla^{2} \tilde{u}-\chi \bar{U} \nabla^{2} \widetilde{v}-\left(3 b_{3} \bar{U}^{2}-2 b_{2} \bar{U}-b_{1}\right) \tilde{u}-\chi \nabla(\widetilde{u} \nabla \widetilde{v}) \\
+\left(b_{2}-3 b_{3} \bar{U}\right) \widetilde{u}^{2}-b_{3} \widetilde{u}^{3}, \\
\widetilde{v}_{t}=D_{v} \nabla^{2} \widetilde{v}+\alpha \tilde{u}-\beta \widetilde{v}, \\
\frac{\partial \widetilde{u}}{\partial x_{i}}=\frac{\partial \widetilde{v}}{\partial x_{i}}=0, \quad \text { at } x_{i}=-\pi, 0, \pi, \text { for } 1 \leq i \leq d,
\end{gathered}
$$

where $[\widetilde{u}(\mathbf{x}, t), \widetilde{v}(\mathbf{x}, t)]$ is the even extension of $[u(\mathbf{x}, t), v(\mathbf{x}, t)]$ on $(-\pi, 0)^{d}$. Taking the second-order derivative of (48) for $x_{i}, x_{j}$ and making inner product with $\partial_{x_{i} x_{j}} \widetilde{u}$ and $A \partial_{x_{i} x_{j}} \widetilde{v}$, respectively, on both sides then adding the two equations together, we have

$$
\begin{aligned}
& \frac{1}{2} \frac{d}{d t} \int_{2 \mathbb{\Psi}^{d}}\left\{\left|\partial_{x_{i} x_{j}} \widetilde{u}\right|^{2}+A\left|\partial_{x_{i} x_{j}} \widetilde{v}\right|^{2}\right\} \mathbf{d} \mathbf{x} \\
& +\int_{2 \mathbb{J}^{d}}\left\{D_{u}\left|\nabla \partial_{x_{i} x_{j}} \widetilde{u}\right|^{2}+A D_{v}\left|\nabla \partial_{x_{i} x_{j}} \widetilde{v}\right|^{2}-\chi \bar{U} \nabla \partial_{x_{i} x_{j}} \tilde{u}\right. \\
& \left.\cdot \nabla \partial_{x_{i} x_{j}} \widetilde{v}\right\} \mathbf{d x}+A \beta \int_{2 \mathbb{T}^{d}}\left|\partial_{x_{i} x_{j}} \widetilde{v}\right|^{2} \mathbf{d} \mathbf{x} \\
& +\left(3 b_{3} \bar{U}^{3}-2 b_{2} \bar{U}-b_{1}\right) \int_{2 \mathbb{T}^{d}}\left|\partial_{x_{i} x_{j}} \tilde{u}\right|^{2} \mathbf{d} \mathbf{x} \\
& =\chi \int_{2 \mathbb{\Psi}^{d}} \nabla \partial_{x_{i} x_{j}} \tilde{u} \cdot \partial_{x_{i} x_{j}}(\widetilde{u} \cdot \nabla \widetilde{v}) \mathbf{d} \mathbf{x}
\end{aligned}
$$

$$
\begin{aligned}
& +A \alpha \int_{2 \mathbb{T}^{d}} \partial_{x_{i} x_{j}} \tilde{u} \cdot \partial_{x_{i} x_{j}} \widetilde{v} \mathbf{d} \mathbf{x}+2\left(b_{2}-3 b_{3} \bar{U}\right) \\
& \times \int_{2 \mathbb{T}^{d}} \partial_{x_{i} x_{j}} \tilde{u}\left(\partial_{x_{i}} \tilde{u} \cdot \partial_{x_{j}} \tilde{u}+\widetilde{u} \cdot \partial_{x_{i} x_{j}} \tilde{u}\right) \mathbf{d} \mathbf{x} \\
& -3 b_{3} \int_{2 \mathbb{J}^{d}} \partial_{x_{i} x_{j}} \tilde{u}\left(2 \tilde{u} \cdot \partial_{x_{i}} \tilde{u} \cdot \partial_{x_{j}} \tilde{u}+\tilde{u}^{2} \cdot \partial_{x_{i} x j} \tilde{u}\right) \mathbf{d} \mathbf{x} \\
& \equiv I_{1}+I_{2}+I_{3}+I_{4} \text {. }
\end{aligned}
$$

Clearly

$$
\begin{gathered}
D_{u}\left|\nabla \partial_{x i, x j} \tilde{u}\right|^{2}+A D_{v}\left|\nabla \partial_{x i, x j} \widetilde{v}\right|^{2}-\chi \bar{U} \nabla \partial_{x_{i} x_{j}} \tilde{u} \cdot \nabla \partial_{x_{i} x_{j}} \widetilde{v} \\
\geq \frac{D_{u}}{2}\left|\nabla \partial_{x_{i} x_{j}} \tilde{u}\right|^{2}+\frac{(\chi \bar{U})^{2}}{2 D_{u}}\left|\nabla \partial_{x_{i} x_{j}} \widetilde{v}\right|^{2} .
\end{gathered}
$$

The nonlinear term $I_{1}$ is bounded by

$$
\begin{gathered}
I_{1} \leq \chi\left\{\|\nabla \widetilde{v}\|_{L^{\infty}}\left\|\nabla \partial_{x_{i} x_{j}} \widetilde{u}\right\|\left\|\partial_{x_{i} x_{j}} \widetilde{u}\right\|+2 \sum_{i=1}^{d}\|\nabla \widetilde{u}\|_{L^{\infty}}\left\|\partial_{x_{i} x_{j}} \widetilde{v}\right\|\right. \\
\left.\times\left\|\nabla \partial_{x_{i} x_{j}} \widetilde{u}\right\|+\|\widetilde{u}\|_{L^{\infty}}\left\|\nabla \partial_{x_{i} x_{j}} \widetilde{u}\right\|\left\|\nabla \partial_{x_{i} x_{j}} \widetilde{v}\right\|\right\} .
\end{gathered}
$$

We know that

$$
\begin{aligned}
\|g\|_{L^{\infty}\left(2 \mathbb{T}^{d}\right)} & \leq E_{6}\|g\|_{H^{2}\left(2 \mathbb{T}^{d}\right)}, \\
\|g\|_{L^{4}\left(2 \mathbb{T}^{d}\right)} & \leq E_{7}\|g\|_{H^{2}\left(2 \mathbb{T}^{d}\right)} . \\
\|g\|_{L^{6}\left(2 \mathbb{T}^{d}\right)} & \leq E_{8}\|g\|_{H^{2}\left(2 \mathbb{T}^{d}\right)},
\end{aligned}
$$

for $d \leq 3$, and

$$
\begin{gathered}
\int_{2 \mathbb{J}^{d}} \nabla \widetilde{u} \mathbf{d} \mathbf{x}=\int_{2 \mathbb{J}^{d}} \nabla \widetilde{v} \mathbf{d} \mathbf{x}=0, \\
\int_{2 \mathbb{J}^{d}} \partial_{x_{i} x_{j}} \widetilde{u} \mathbf{d} \mathbf{x}=\int_{2 \mathbb{T}^{d}} \partial_{x_{i} x_{j}} \widetilde{v} \mathbf{d} \mathbf{x}=0 .
\end{gathered}
$$

Applying the Poincaré inequality, we have

$$
\|g\| \leq E_{9}\|g\|_{L^{4}\left(2 \mathbb{T}^{d}\right)} \leq E_{10}\|g\|_{H^{1}} \leq E_{11}\|\nabla g\|, \quad d \leq 3 .
$$

It follows from (53) and (54) that $\left\|\partial_{x_{i}} g\right\| \leq E_{11}\left\|\nabla \partial_{x_{i}} g\right\|$. Thus

$$
\|\nabla g\| \leq E_{11}\left(\sum_{i, j=1}^{d}\left\|\partial_{x_{i} x_{j}} g\right\|^{2}\right)^{1 / 2}
$$

Furthermore,

$$
\frac{1}{d^{2}} \sum_{i, j=1}^{d}\left\|\partial_{x_{i} x_{j}} g\right\|^{2} \leq \sum_{|\alpha|=2}\left\|D^{\alpha} g\right\|^{2} \leq \sum_{i, j=1}^{d}\left\|\partial_{x_{i} x_{j}} g\right\|^{2} .
$$


This implies that $\left(\sum_{i, j=1}^{d}\left\|\partial_{x_{i} x_{j}} g\right\|^{2}\right)^{1 / 2}$ is equivalent to $\left(\sum_{|\alpha|=2}\left\|D^{\alpha} g\right\|^{2}\right)^{1 / 2}$. From (53)-(56), we have

$$
\begin{gathered}
\left\|\partial_{x_{i} x_{j}} g\right\| \leq E_{11}\left\|\nabla \partial_{x_{i} x_{j}} g\right\|, \\
\|\nabla g\| \leq E_{11} d\left(\sum_{|\alpha|=2}\left\|D^{\alpha} g\right\|^{2}\right)^{1 / 2} \\
\leq E_{11}^{2} d\left(\sum_{|\alpha|=2}\left\|\nabla D^{\alpha} g\right\|^{2}\right)^{1 / 2} .
\end{gathered}
$$

It follows from (56) and (57) that

$$
\begin{gathered}
\|\nabla g\|_{H^{2}} \leq E_{12}\left(\sum_{|\alpha|=2}\left\|\nabla D^{\alpha} g\right\|^{2}\right)^{1 / 2}, \\
E_{12}=\left(E_{11}^{4} d^{2}+E_{11}^{2} d^{2}+1\right)^{1 / 2} .
\end{gathered}
$$

By (51), (52), (57), and (58), we have

$$
\sum_{|\alpha|=2} I_{1} \leq \chi E_{13}\|\widetilde{\mathbf{w}}\|_{H^{2}}\left\|\nabla^{3} \widetilde{\mathbf{w}}\right\|^{2}
$$

Now we consider $I_{2}$. From Gagliardo-Nirenberg inequality and Young inequality, we obtain

$$
\left\|\partial_{x_{i} x_{j}} \widetilde{u}\right\|^{2} \leq \frac{8 E_{14}^{2}}{9}\left(a\left\|\nabla \partial_{x_{i} x_{j}} \widetilde{u}\right\|^{2}+\frac{\|\tilde{u}\|^{2}}{4 a^{2}}\right) .
$$

Let $a=9 D_{u}^{3} D_{v}^{2} \beta^{2}\left(3 b_{3} \bar{U}^{2}-2 b_{2} \bar{U}-b_{1}\right) / 4 E_{14}^{2} \chi^{4} \bar{U}^{4} \alpha^{4}$. Then

$$
\begin{aligned}
\sum_{|\alpha|=2} I_{2} \leq & \frac{3 b_{3} \bar{U}^{2}-2 b_{2} \bar{U}-b_{1}}{2} \sum_{|\alpha|=2}\left\|D^{\alpha} \widetilde{u}\right\|^{2} \\
& +\frac{A \beta}{2} \sum_{|\alpha|=2}\left\|D^{\alpha} \widetilde{v}\right\|^{2}+\frac{D_{u}}{4} \sum_{|\alpha|=2}\left\|\nabla D^{\alpha} \widetilde{u}\right\|^{2} \\
& +\frac{E_{15} \chi^{12} \bar{U}^{12} \alpha^{12}}{D_{u}^{8} D_{v}^{6} \beta^{6}\left(3 b_{3} \bar{U}^{2}-2 b_{2} \bar{U}-b_{1}\right)^{3}}\|\widetilde{u}\|^{2} .
\end{aligned}
$$

It follows from (52), (57), and (58) that

$$
\begin{gathered}
\sum_{|\alpha|=2} I_{3} \leq 2\left|b_{2}-3 b_{3} \bar{U}\right| E_{16}\|\widetilde{\mathbf{w}}\|_{H^{2}}\left\|\nabla^{3} \widetilde{\mathbf{w}}\right\|^{2}, \\
\sum_{|\alpha|=2} I_{4} \leq 3 b_{3} E_{17}\|\widetilde{\mathbf{w}}\|^{2}\left\|\nabla^{3} \widetilde{\mathbf{w}}\right\| .
\end{gathered}
$$

Combining (49), (50), (59), (61), and (62), we have

$$
\begin{aligned}
\frac{1}{2} \frac{d}{d t} \sum_{|\alpha|=2} & \int_{\mathbb{T}^{d}}\left\{\left|D^{\alpha} u\right|^{2}+\frac{(\chi \bar{U})^{2}}{D_{u} D_{v}}\left|D^{\alpha} v\right|^{2}\right\} \mathbf{d x} \\
& +\sum_{|\alpha|=2} \int_{\mathbb{T}^{d}}\left\{\frac{D_{u}}{4}\left|\nabla D^{\alpha} u\right|^{2}+\frac{(\chi \bar{U})^{2}}{2 D_{u}}\left|\nabla D^{\alpha} v\right|^{2}\right\} \mathbf{d} \mathbf{x} \\
& +\frac{A \beta}{2} \sum_{|\alpha|=2} \int_{\mathbb{T}^{d}}\left|D^{\alpha} v\right|^{2} \mathbf{d x}+\frac{3 b_{3} \bar{U}^{2}-2 b_{2} \bar{U}-b_{1}}{2} \\
& \times \sum_{|\alpha|=2} \int_{\mathbb{T}^{d}}\left|D^{\alpha} u\right|^{2} \mathbf{d x} \leq C_{0} \frac{\chi+2\left|b_{2}-3 b_{3} \bar{U}\right|+3 b_{3}}{2} \\
& \times\left(\|\mathbf{w}\|_{H^{2}}+\|\mathbf{w}\|_{H^{2}}^{2}\right)\left\|\nabla^{3} \mathbf{w}\right\|^{2}+C_{2}\|u\|^{2},
\end{aligned}
$$

where $C_{0}=\max \left\{E_{13}, E_{16}, E_{17}\right\}, C_{2}=E_{15} \chi^{12} \bar{U}^{12} \alpha^{12} / 2 D_{u}^{8} D_{v}^{6}$ $\beta^{6}\left(3 b_{3} \bar{U}^{2}-2 b_{2} \bar{U}-b_{1}\right)^{3}$.

Lemma 6. Let $\mathbf{w}(\mathbf{x}, t)$ be a solution of (18) such that for $0 \leq$ $t \leq T<T_{0}$

$$
\begin{aligned}
&\|\mathbf{w}(\cdot, t)\|_{H^{2}}+\|\mathbf{w}(\cdot, t)\|_{H^{2}}^{2} \\
& \leq \frac{1}{C_{0}} \min \left\{\frac{D_{u}}{2\left(\chi+2\left|b_{2}-3 b_{3} \bar{U}\right|+3 b_{3}\right)},\right. \\
&\left.\frac{(\chi \bar{U})^{2}}{D_{u}\left(\chi+2\left|b_{2}-3 b_{3} \bar{U}\right|+3 b_{3}\right)}\right\},
\end{aligned}
$$

$$
\|\mathbf{w}(\cdot, t)\| \leq 2 C_{1} \exp \left(\lambda_{\max } t\right)\|\mathbf{w}(\cdot, 0)\| .
$$

Then

$$
\begin{aligned}
\|\mathbf{w}(\cdot, t)\|_{H^{2}}^{2} \leq C_{3}\{ & \left\{\|\mathbf{w}(\cdot, 0)\|_{H^{2}}^{2}\right. \\
& \left.+\exp \left(2 \lambda_{\max } t\right)\|\mathbf{w}(\cdot, 0)\|^{2}\right\}, \quad 0 \leq t \leq T,
\end{aligned}
$$

where $C_{3}=\max \left\{\left(E_{11}^{2} d^{2}+1\right)\left((\chi \bar{U})^{2} / D_{u} D_{v}\right), 4 C_{1}^{2}\{1+\right.$ $\left.\left.\left(E_{11}^{2} d^{2}+1\right)\left(C_{2} / \lambda_{\max }\right)\right\}\right\}$ if $(\chi \bar{U})^{2} / D_{u} D_{v} \geq 1, C_{3}=$ $\max \left\{\left(E_{11}^{2} d^{2}+1\right)\left(D_{u} D_{v} /(\chi \bar{U})^{2}\right), 4 C_{1}^{2}\left\{1+\left(E_{11}^{2} d^{2}+1\right)\right.\right.$ $\left.\left.\left(C_{2} D_{u} D_{v} / \lambda_{\max }(\chi \bar{U})^{2}\right)\right\}\right\}$ if $(\chi \bar{U})^{2} / D_{u} D_{v}<1$.

Proof. It is clear from (57) that

$$
\|\nabla \mathbf{w}(\cdot, t)\|^{2} \leq E_{11}^{2} d^{2} \sum_{|\alpha|=2}\left\|D^{\alpha} \mathbf{w}(\cdot, t)\right\|^{2} .
$$

It follows from (67) that

$$
\|\mathbf{w}(\cdot, t)\|_{H^{2}}^{2} \leq\|\mathbf{w}(\cdot, t)\|^{2}+\left(E_{11}^{2} d^{2}+1\right) \sum_{|\alpha|=2}\left\|D^{\alpha} \mathbf{w}(\cdot, t)\right\|^{2} .
$$


Now we estimate the second-order derivatives of $\mathbf{w}(\cdot, t)$. By (65) and Lemma 5, we immediately see that

$$
\begin{gathered}
\frac{1}{2} \frac{d}{d t} \sum_{|\alpha|=2} \int_{\mathbb{T}^{d}}\left\{\left|D^{\alpha} u\right|^{2}+\frac{(\chi \bar{U})^{2}}{D_{u} D_{v}}\left|D^{\alpha} v\right|^{2}\right\} \mathbf{d x} \\
\leq C_{2}\|u\|^{2} \leq C_{2}\|\mathbf{w}(\cdot, t)\|^{2} .
\end{gathered}
$$

Integrating on both sides of (69) from 0 to $t$ and from (65), we have

$$
\begin{aligned}
& \sum_{|\alpha|=2} \int_{\mathbb{T}^{d}}\left\{\left|D^{\alpha} u(\cdot, t)\right|^{2}+\frac{(\chi \bar{U})^{2}}{D_{u} D_{v}}\left|D^{\alpha} v(\mathbf{x}, t)\right|^{2}\right\} \mathbf{d x} \\
& \leq \sum_{|\alpha|=2} \int_{\mathbb{T}^{d}}\left\{\left|D^{\alpha} u(\cdot, 0)\right|^{2}+\frac{(\chi \bar{U})^{2}}{D_{u} D_{v}}\left|D^{\alpha} v(\cdot, 0)\right|^{2}\right\} \mathbf{d x} \\
& +\frac{4 C_{1}^{2} C_{2}}{\lambda_{\max }}\|\mathbf{w}(\cdot, 0)\|^{2} \exp \left(2 \lambda_{\max } t\right) .
\end{aligned}
$$

We will proceed in the following two cases: $(\chi \bar{U})^{2} / D_{u} D_{v} \geq 1$, $(\chi \bar{U})^{2} / D_{u} D_{v}<1$.

(1) If $(\chi \bar{U})^{2} / D_{u} D_{v} \geq 1$, it follows from (70) that

$$
\begin{gathered}
\sum_{|\alpha|=2}\left\|D^{\alpha} \mathbf{w}(\cdot, t)\right\|^{2} \leq \frac{(\chi \bar{U})^{2}}{D_{u} D_{v}} \sum_{|\alpha|=2}\left\|D^{\alpha} \mathbf{w}(\cdot, 0)\right\|^{2} \\
+\frac{4 C_{1}^{2} C_{2}}{\lambda_{\max }}\|\mathbf{w}(\cdot, 0)\|^{2} \exp \left(2 \lambda_{\max } t\right) .
\end{gathered}
$$

By (68) and (71), we have

$$
\|\mathbf{w}(\cdot, t)\|_{H^{2}}^{2} \leq C_{3}\left\{\|\mathbf{w}(\cdot, 0)\|_{H^{2}}^{2}+\|\mathbf{w}(\cdot, 0)\|^{2} \exp \left(2 \lambda_{\max } t\right)\right\},
$$

where $C_{3}=\max \left\{\left(E_{11}^{2} d^{2}+1\right)\left((\chi \bar{U})^{2} / D_{u} D_{v}\right), 4 C_{1}^{2}\left\{1+\left(E_{11}^{2} d^{2}+\right.\right.\right.$ 1) $\left.\left.\left(C_{2} / \lambda_{\max }\right)\right\}\right\}$.

(2) If $(\chi \bar{U})^{2} / D_{u} D_{v}<1$, it follows from (71) that

$$
\begin{aligned}
\sum_{|\alpha|=2}\left\|D^{\alpha} \mathbf{w}(\cdot, t)\right\|^{2} & \leq \frac{D_{u} D_{v}}{(\chi \bar{U})^{2}} \sum_{|\alpha|=2}\left\|D^{\alpha} \mathbf{w}(\cdot, 0)\right\|^{2} \\
+ & \frac{D_{u} D_{v}}{(\chi \bar{U})^{2}} \cdot \frac{4 C_{1}^{2} C_{2}}{\lambda_{\max }}\|\mathbf{w}(\cdot, 0)\|^{2} \exp \left(2 \lambda_{\max } t\right) .
\end{aligned}
$$

By (68) and (73), we have

$$
\|\mathbf{w}(\cdot, t)\|_{H^{2}}^{2} \leq C_{3}\left\{\|\mathbf{w}(\cdot, 0)\|_{H^{2}}^{2}+\|\mathbf{w}(\cdot, 0)\|^{2} \exp \left(2 \lambda_{\max } t\right)\right\},
$$

where $C_{3}=\max \left\{\left(E_{11}^{2} d^{2}+1\right)\left(D_{u} D_{v} /(\chi \bar{U})^{2}\right), 4 C_{1}^{2}\left\{1+\left(E_{11}^{2} d^{2}+\right.\right.\right.$ 1) $\left.\left.\left(C_{2} D_{u} D_{v} / \lambda_{\max }(\chi \bar{U})^{2}\right)\right\}\right\}$.

\section{Main Result}

Let $\theta$ be a small fixed constant, and $\lambda_{\max }$ be the dominant eigenvalue which is the maximal growth rate. We also denote the gap between the largest growth rate $\lambda_{\max }$ and the rest by $v>0$. Then for $\delta>0$ arbitrary small, we define the escape time $T^{\delta}$ by

$$
\theta=\delta \exp \left(\lambda_{\max } T^{\delta}\right)
$$

or equivalently

$$
T^{\delta}=\frac{1}{\lambda_{\max }} \ln \frac{\theta}{\delta} .
$$

Our main result is as follows.

Theorem 7. Assume that the set of $q^{2}=\sum_{i=1}^{d} q_{i}^{2}$ satisfying instability criterion (22) is not empty for given parameters $D_{u}$, $\chi, \bar{U}, b_{1}, b_{2}, b_{3}, D_{v}, \alpha, \beta$. Let

$$
\mathbf{w}_{0}(\mathbf{x})=\sum_{\mathbf{q} \in \Omega}\left\{w_{\mathbf{q}}^{-} \mathbf{r}_{-}(\mathbf{q})+w_{\mathbf{q}}^{+} \mathbf{r}_{+}(\mathbf{q})\right\} e_{\mathbf{q}}(\mathbf{x}) \in H^{2}
$$

such that $\left\|\mathbf{w}_{0}\right\|=1$. Then there exist constants $\delta_{0}>0, C>0$ and $\theta>0$ depending on $D_{u}, \chi, \bar{U}, b_{1}, b_{2}, b_{3}, D_{v}, \alpha$, and $\beta$, such that for all $0<\delta \leq \delta_{0}$, if the initial perturbation of the steady state $[\bar{U}, \bar{V}]$ is $\mathbf{w}^{\delta}(\cdot, 0)=\delta \mathbf{w}_{0}$, then its nonlinear evolution $\mathbf{w}^{\delta}(\cdot, t)$ satisfies

$$
\begin{gathered}
\left\|\mathbf{w}^{\delta}(\cdot, t)-\delta e^{\lambda_{\text {max }} t} \sum_{q \in \Omega_{\max }} w_{\mathbf{q}}^{+} \mathbf{r}_{+}(\mathbf{q}) e_{\mathbf{q}}(\mathbf{x})\right\| \\
\leq C\left\{e^{-v t}+\delta\left\|\mathbf{w}_{0}\right\|_{H^{2}}^{2}+\delta^{2}\left\|\mathbf{w}_{0}\right\|_{H^{2}}^{3}+\delta e^{\lambda_{\max }{ }^{t}}\right. \\
\left.+\delta^{2} e^{2 \lambda_{\max } t}\right\} \delta e^{\lambda_{\max } t}
\end{gathered}
$$

for $0 \leq t \leq T^{\delta}$, and $v>0$ is the gap between $\lambda_{\max }$ and the rest of $\lambda_{\mathbf{q}}$ in (21).

Proof. Let $\mathbf{w}^{\delta}(\mathbf{x}, t)$ be the solutions to (18) with initial data $\mathbf{w}^{\delta}(\cdot, 0)=\delta \mathbf{w}_{0}$. We define

$$
T^{*}=\sup \left\{t \mid\left\|\mathbf{w}^{\delta}(\cdot, t)-\delta e^{\mathfrak{\Omega} t} \mathbf{w}_{0}\right\| \leq \frac{C_{1}}{2} \delta \exp \left(\lambda_{\max } t\right)\right\} .
$$

We also define

$T^{* *}=\sup$

$\times\left\{t \mid \leq \frac{1}{C_{0}^{*}} \min \left\{\frac{\left\|\mathbf{w}^{\delta}(\cdot, t)\right\|_{H^{2}}+\left\|\mathbf{w}^{\delta}(\cdot, t)\right\|_{H^{2}}^{2}}{2\left(\chi+2\left|b_{2}-3 b_{3} \bar{U}\right|+3 b_{3}\right)}, \frac{(\chi \bar{U})^{2}}{D_{u}\left(\chi+2\left|b_{2}-3 b_{3} \bar{U}\right|+3 b_{3}\right)}\right\}\right\}$. 
Choose $\theta$ such that

$$
\begin{aligned}
& C_{0}^{*} C_{3} \theta\left(1+2 C_{3}^{1 / 2} \theta\right) \\
&<\min \left\{\frac{\lambda_{\max }}{4\left(\chi+\left|b_{2}-3 b_{3} \bar{U}\right|+b_{3}\right)},\right. \\
& \frac{D_{u}}{4\left(\chi+2\left|b_{2}-3 b_{3} \bar{U}\right|+3 b_{3}\right)}, \\
&\left.\frac{(\chi \bar{U})^{2}}{2 D_{u}\left(\chi+2\left|b_{2}-3 b_{3} \bar{U}\right|+3 b_{3}\right)}\right\} .
\end{aligned}
$$

We now establish a sharper $L^{2}$ estimate of $\mathbf{w}^{\delta}(\cdot, t)$ for $0 \leq$ $t \leq \min \left\{T^{\delta}, T^{*}, T^{* *}\right\}$. First of all, by the definition of $T^{*}$ and Lemma 2 , for $0<t \leq T^{*}$, it is not hard to see that

$$
\left\|\mathbf{w}^{\delta}(\cdot, t)\right\| \leq \frac{3}{2} C_{1} \delta \exp \left(\lambda_{\max } t\right) .
$$

Applying Lemma 6 and the bootstrap argument, one can prove

$$
\left\|\mathbf{w}^{\delta}(\cdot, t)\right\|_{H^{2}} \leq \sqrt{C_{3}}\left\{\delta\left\|\mathbf{w}_{0}\right\|_{H^{2}}+\delta \exp \left(\lambda_{\max } t\right)\right\} .
$$

From this and $(a+b)^{p} \leq 2^{p-1}\left(a^{p}+b^{p}\right)(a \geq 0, b \geq 0, p \geq 1)$, it follows that

$$
\left\|\mathbf{w}^{\delta}(\cdot, t)\right\|_{H^{2}}^{3} \leq 4 C_{3}^{3 / 2}\left\{\delta^{3}\left\|\mathbf{w}_{0}\right\|_{H^{2}}^{3}+\delta^{3} \exp \left(3 \lambda_{\max } t\right)\right\} .
$$

Applying Duhamel's principle, we can obtain

$$
\begin{aligned}
\mathbf{w}^{\delta}(\cdot, t)=\delta e^{\mathfrak{Q t}} \mathbf{w}_{0} & \\
-\int_{0}^{t} e^{\mathcal{Q}(t-\tau)} & {\left[\chi \nabla\left(u^{\delta}(\tau) \nabla v^{\delta}(\tau)\right)\right.} \\
& -\left(b_{2}-3 b_{3} \bar{U}\right)\left(u^{\delta}(\tau)\right)^{2} \\
& \left.+b_{3}\left(u^{\delta}(\tau)\right)^{3}, 0\right] d \tau .
\end{aligned}
$$

By Lemma 2, (52), (54), and Lemma 6, for $0 \leq t \leq$ $\min \left\{T^{\delta}, T^{*}, T^{* *}\right\}$, we deduce that

$$
\begin{array}{r}
\left\|\mathbf{w}^{\delta}(\cdot, t)-\delta e^{\Omega t} \mathbf{w}_{0}\right\| \leq C_{1} C_{0}^{*}\left\{\chi+\left|b_{2}-3 b_{3} \bar{U}\right|+b_{3}\right\} \\
\times \int_{0}^{t} e^{\lambda_{\max }(t-\tau)}\left\{\left\|\mathbf{w}^{\delta}(\tau)\right\|_{H^{2}}^{2}+\left\|\mathbf{w}^{\delta}(\tau)\right\|_{H^{2}}^{3}\right\} d \tau,
\end{array}
$$

where $C_{0}^{*}=\max \left\{E_{7}^{2},\left(E_{11}^{2} / E_{9}^{2}\right)+E_{6}, E_{8}^{3}\right\}$. By our choice of $t \leq$ $\min \left\{T^{\delta}, T^{*}, T^{* *}\right\}$, it is further bounded by

$$
\begin{aligned}
& \| \mathbf{w}^{\delta}(t)- \delta e^{\mathcal{Q} t} \mathbf{w}_{0} \| \\
& \leq C_{1} C_{0}^{*} C_{3}\left(\chi+\left|b_{2}-3 b_{3} \bar{U}\right|+b_{3}\right) \\
& \times \frac{\delta\left\|\mathbf{w}_{0}\right\|_{H^{2}}^{2}+4 C_{3}^{1 / 2} \delta^{2}\left\|\mathbf{w}_{0}\right\|_{H^{2}}^{3}}{\lambda_{\max }} \\
&\left.+\frac{\delta e^{\lambda_{\max } t}+2 C_{3}^{1 / 2} \delta^{2} e^{2 \lambda_{\max } t}}{\lambda_{\max }}\right\} \delta e^{\lambda_{\max } t} .
\end{aligned}
$$

We now prove by contradiction that for $\delta$ sufficiently small, $T^{\delta}=\min \left\{T^{\delta}, T^{*}, T^{* *}\right\}$. If $T^{* *}$ is the smallest, we can let $t=$ $T^{* *} \leq T^{\delta}$ in (83) and (84). If $\theta$ satisfies (81) with $C_{3} \geq 1$ and $\delta$ is sufficiently small such that $C_{3} \delta^{2}\left\|\mathbf{w}_{0}\right\|_{H^{2}}^{2}+\sqrt{C_{3}} \delta\left\|\mathbf{w}_{0}\right\|_{H^{2}} \leq$ $\left(1 / 2 C_{0}^{*}\right) \min \left\{D_{u} /\left(2\left(\chi+2\left|b_{2}-3 b_{3} \bar{U}\right|+3 b_{3}\right)\right),(\chi \bar{U})^{2} / D_{u}(\chi+\right.$ $\left.\left.2\left|b_{2}-3 b_{3} \bar{U}\right|+3 b_{3}\right)\right\}$, we immediately see that

$$
\begin{gathered}
\left\|\mathbf{w}^{\delta}\left(T^{* *}\right)\right\|_{H^{2}}+\left\|\mathbf{w}^{\delta}\left(T^{* *}\right)\right\|_{H^{2}}^{2} \\
\leq C_{3} \delta^{2}\left\|\mathbf{w}_{0}\right\|_{H^{2}}^{2}+\sqrt{C_{3}} \delta\left\|\mathbf{w}_{0}\right\|_{H^{2}}+C_{3}^{1 / 2} \theta\left(1+C_{3}^{1 / 2} \theta\right) \\
<\frac{1}{C_{0}^{*}} \min \left\{\frac{D_{u}}{2\left(\chi+2\left|b_{2}-3 b_{3} \bar{U}\right|+3 b_{3}\right)},\right. \\
\left.\frac{(\chi \bar{U})^{2}}{D_{u}\left(\chi+2\left|b_{2}-3 b_{3} \bar{U}\right|+3 b_{3}\right)}\right\} .
\end{gathered}
$$

This is a contradiction to the definition of $T^{* *}$. On the other hand, if $T^{*}$ is the smallest, we can let $t=T^{*}$ in (87). If $\theta$ satisfies (81) and $\delta$ is sufficiently small such that $C_{0}^{*} C_{3}(\chi+$ $\left.\left|b_{2}-3 b_{3} \bar{U}\right|+b_{3}\right)\left(\delta\left\|\mathbf{w}_{0}\right\|_{H^{2}}^{2}+4 C_{3}^{1 / 2} \delta^{2}\left\|\mathbf{w}_{0}\right\|_{H^{2}}^{3} / \lambda_{\max }\right)<1 / 4$, we also can see that

$$
\begin{aligned}
& \left\|\mathbf{w}^{\delta}\left(\cdot, T^{*}\right)-\delta e^{\mathfrak{R} T^{*}} \mathbf{w}_{0}\right\| \\
& \leq C_{1} C_{0}^{*} C_{3}\left(\chi+\left|b_{2}-3 b_{3} \bar{U}\right|+b_{3}\right) \\
& \times\left\{\frac{\delta\left\|\mathbf{w}_{0}\right\|_{H^{2}}^{2}+4 C_{3}^{1 / 2} \delta^{2}\left\|\mathbf{w}_{0}\right\|_{H^{2}}^{3}}{\lambda_{\max }}\right. \\
& \left.+\frac{\theta\left(1+2 C_{3}^{1 / 2} \theta\right)}{\lambda_{\max }}\right\} \delta e^{\lambda_{\max } T^{*}}<\frac{C_{1}}{2} \delta e^{\lambda_{\max } T^{*}} .
\end{aligned}
$$

This again contradicts the definition of $T^{*}$. Hence, if $\delta$ is sufficiently small, we have

$$
T^{\delta}=\min \left\{T^{\delta}, T^{*}, T^{* *}\right\}
$$


From (28), we have

$$
\begin{aligned}
\| \mathbf{w}^{\delta}(\cdot, t) & -\delta e^{\Omega t} \mathbf{w}_{0} \| \\
\geq & \left\|\mathbf{w}^{\delta}(\cdot, t)-\delta e^{\lambda_{\max } t} \sum_{\mathbf{q} \in \Omega_{\max }} w_{\mathbf{q}}^{+} \mathbf{r}_{+}(\mathbf{q}) e_{\mathbf{q}}(\mathbf{x})\right\| \\
& -\left\|\delta \sum_{\mathbf{q} \in \Omega_{\max }} w_{\mathbf{q}}^{-} \mathbf{r}_{-}(\mathbf{q}) \exp \left(\lambda_{\mathbf{q}}^{-} t\right) e_{\mathbf{q}}(\mathbf{x})\right\| \\
& -\| \sum_{\mathbf{q} \in \Omega \backslash \Omega_{\max }}\left\{w_{\mathbf{q}}^{-} \mathbf{r}_{-}(\mathbf{q}) \exp \left(\lambda_{\mathbf{q}}^{-} t\right)\right. \\
\equiv & \left\|\mathbf{w}^{\delta}(\cdot, t)-\delta e^{\lambda_{\max } t} \sum_{\mathbf{q} \in \Omega_{\max }} w_{\mathbf{q}}^{+} \mathbf{r}_{+}(\mathbf{q}) e_{\mathbf{q}}(\mathbf{x})\right\|-I_{1}-I_{2} ;
\end{aligned}
$$

that is,

$$
\begin{gathered}
\left\|\mathbf{w}^{\delta}(\cdot, t)-\delta e^{\lambda_{\max } t} \sum_{\mathbf{q} \in \Omega_{\max }} w_{\mathbf{q}}^{+} \mathbf{r}_{+}(\mathbf{q}) e_{\mathbf{q}}(\mathbf{x})\right\| \\
\leq\left\|\mathbf{w}^{\delta}(\cdot, t)-\delta e^{\mathfrak{Q} t} \mathbf{w}_{0}\right\|+I_{1}+I_{2} .
\end{gathered}
$$

Using (33) and (34), we have

$$
I_{1}^{2} \leq \delta^{2} e^{2\left(\lambda_{\max }-v\right) t}\left(\frac{\pi}{2}\right)^{d} \sum_{\mathbf{q} \in \Omega_{\max }} E_{2}^{2} E_{3}^{2} q^{6}\left|\mathbf{w}_{q}\right|^{2} .
$$

We know that there is one (or two) $q^{2}$ satisfying $\lambda^{+}\left(q^{2}\right)=$ $\lambda_{\text {max }}$. If there is only one $q^{2}$ satisfying $\lambda^{+}\left(q^{2}\right)=\lambda_{\max }$, we denote it by $q_{\max }^{2}$. If there are $q_{1}^{2}$ and $q_{2}^{2}$ satisfying $\lambda^{+}\left(q^{2}\right)=$ $\lambda_{\text {max }}$, we let $q_{\max }^{2}=\max \left\{q_{1}^{2}, q_{2}^{2}\right\}$. From (93), we have

$$
I_{1} \leq E_{2} E_{3} q_{\max }^{3} \delta e^{\left(\lambda_{\max }-v\right) t}=C^{*} \delta e^{\left(\lambda_{\max }-v\right) t}
$$

where $C^{*}=E_{2} E_{3} q_{\text {max }}^{3}$. Now we consider $I_{2}$. By (38), we have

$$
I_{2} \leq \delta e^{\left(\lambda_{\max }-v\right) t}
$$

From (87), (92), (94), and (95), it follows that

$$
\begin{array}{r}
\left\|\mathbf{w}^{\delta}(\cdot, t)-\delta e^{\lambda_{\max } t} \sum_{\mathbf{q} \in \Omega_{\max }} w_{\mathbf{q}}^{+} \mathbf{r}_{+}(\mathbf{q}) e_{\mathbf{q}}(\mathbf{x})\right\| \\
\leq C\left\{e^{-v t}+\delta\left\|\mathbf{w}_{0}\right\|_{H^{2}}^{2}+\delta^{2}\left\|\mathbf{w}_{0}\right\|_{H^{2}}^{3}\right. \\
\left.+\delta e^{\lambda_{\max } t}+\delta^{2} e^{2 \lambda_{\max } t}\right\} \delta e^{\lambda_{\text {max }} t},
\end{array}
$$

where $C=\max \left\{C^{*}+1,\left(4 C_{1} C_{0}^{*} C_{3}^{3 / 2} / \lambda_{\max }\right)\left(\chi+\left|b_{2}-3 b_{3} \bar{U}\right|+\right.\right.$ $\left.\left.b_{3}\right)\right\}$. Notice that for $0 \leq t \leq T^{\delta}, \delta e^{\lambda_{\max } t} \leq \theta$ is sufficiently small. As long as $w_{\mathbf{q}_{0}}^{+} \neq 0$ for at least one $\mathbf{q}_{0} \in \Omega_{\max }$, which is generic for perturbations, the corresponding fastest growing modes

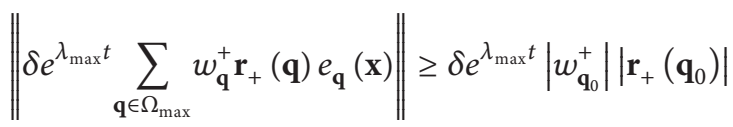

have the dominant leading order of $\delta e^{\lambda_{\max } t}$.

Our Theorem 7 implies that the dynamics of a general perturbation is characterized by such linear dynamics over a long time period of $\varepsilon T^{\delta} \leq t \leq T^{\delta}$, for any $\varepsilon>0$. In particular, choose a fixed $\mathbf{q}_{0}=\left(q_{01}, q_{02}, \ldots, q_{0 d}\right) \in \Omega_{\max }$ and let $\mathbf{w}_{0}(\mathbf{x})=\mathbf{r}_{+}\left(\mathbf{q}_{0}\right) /\left|\mathbf{r}_{+}\left(\mathbf{q}_{0}\right)\right| e_{\mathbf{q}_{0}}(\mathbf{x})$; then

$$
\left\|\mathbf{w}_{0}(\mathbf{x})\right\|_{H^{2}}=\left\{\left(\frac{\pi}{2}\right)^{d}\left(1+\left|\mathbf{q}_{0}\right|^{2}+\left|\mathbf{q}_{0}\right|^{4}\right)\right\}^{1 / 2} .
$$

Note that $\delta \leq \theta, \theta$, and $v$ are fixed constants and $\mathbf{q}_{0}$ is a fixed vector. From (96) and (98), if $t \leq T^{\delta}$, we have

$$
\begin{gathered}
\left\|\mathbf{w}^{\delta}(\cdot, t)-\delta e^{\lambda_{\max } T^{\delta}} \frac{\mathbf{r}_{+}\left(\mathbf{q}_{0}\right)}{\left|\mathbf{r}_{+}\left(\mathbf{q}_{0}\right)\right|} e_{\mathbf{q}_{0}}(\mathbf{x})\right\| \\
\leq C^{* *}\left\{\delta^{\nu / \lambda_{\max }}+\theta^{2}+\theta^{3}\right\},
\end{gathered}
$$

where $C^{* *}=C C_{4}, C_{4}=\max \left\{\theta^{1-\left(\nu / \lambda_{\max }\right)},(\pi / 2)^{3 d / 2}\left(1+\left|\mathbf{q}_{0}\right|^{2}+\right.\right.$ $\left.\left.\left|\mathbf{q}_{0}\right|^{4}\right)^{3 / 2}+1\right\}$. Moreover,

$$
\left\|\mathbf{w}^{\delta}(\cdot, t)\right\| \geq \theta-C^{* *}\left\{\delta^{\nu / \lambda_{\max }}+\theta^{2}+\theta^{3}\right\} .
$$

Let $0<\theta<\left(\sqrt{1+\left(2 / C^{* *}\right)}-1\right) / 2$, and $\delta_{0}=$ $\left(\left(\theta / 2 C^{* *}\right)-\theta^{2}-\theta^{3}\right)^{\lambda_{\max } / v}$. Then

$$
\left\|\mathbf{w}^{\delta}(\cdot, t)\right\| \geq \theta-C^{* *}\left\{\delta^{\nu / \lambda_{\max }}+\theta^{2}+\theta^{3}\right\} \geq \frac{\theta}{2}>0, \quad 0<\delta \leq \delta_{0} .
$$

This implies nonlinear instability as $\delta \rightarrow 0$. In particular, instability occurs before the possible blow-up time.

Let us point out that although our proof is based on GuoStrauss' bootstrap argument, the adaptation to the procedure to our problem is not trivial at all, since the appearance of a growth restriction of a cubic type, we need more delicate estimates. Notice in our theorem that each initial perturbation can be drastically different from another, which gives rise to the richness of the pattern; on the other hand, the finite number maximal growing modes determines the common characteristics of the pattern, over the time scale of $\ln (1 / \delta)$. Therefore, our result indeed provides a mathematical description for the pattern formation in the Keller-Segel model with a cubic source term.

\section{Acknowledgments}

The authors would like to thank the referees for their helpful comments. This work is supported by the China National Natural Science Foundation (nos. 11061031; 11261053), the Fundamental Research Funds for the Gansu University. 


\section{References}

[1] E. Keller and L. Segel, "Initiation of Slime mold aggregation viewed as an instability," Journal of Theoretical Biology, vol. 26, no. 3, pp. 399-415, 1970.

[2] D. Horstmann, "From 1970 until present: the Keller-Segel model in chemotaxis and its consequences. I," Jahresbericht der Deutschen Mathematiker-Vereinigung, vol. 105, no. 3, pp. 103165, 2003.

[3] D. Horstmann, "From 1970 until present: the Keller-Segel model in chemotaxis and its consequences. II," Jahresbericht der Deutschen Mathematiker-Vereinigung, vol. 106, no. 2, pp. 51-69, 2004.

[4] D. Horstmann, "Generalizing the Keller-Segel model: Lyapunov functionals, steady state analysis, and blow-up results for multispecies chemotaxis models in the presence of attraction and repulsion between competitive interacting species," Journal of Nonlinear Science, vol. 21, no. 2, pp. 231-270, 2011.

[5] Y. Guo and H. J. Hwang, "Pattern formation (I): the Keller-Segel model," Journal of Differential Equations, vol. 249, no. 7, pp. 15191530, 2010.

[6] K. Painter and T. Hillen, "Spatio-temporal chaos in a chemotaxis model," Physica D, vol. 240, no. 4-5, pp. 363-375, 2011.

[7] S. Banerjee, A. Misra, and L. Rondoni, "Spatiotemporal evolution in a $(2+1)$-dimensional chemotaxis model," Physica A, vol. 391, no. 1-2, pp. 107-122, 2012.

[8] K. Kuto, K. Osaki, T. Sakurai, and T. Tsujikawa, "Spatial pattern formation in a chemotaxisdiffusion-growth model," Physica D, vol. 241, no. 19, pp. 1629-1639, 2012.

[9] T. Okuda and K. Osaki, "Bifurcation of hexagonal patterns in a chemotaxis-diffusion-growth system," Nonlinear Analysis: Real World Applications, vol. 12, no. 6, pp. 3294-3305, 2011.

[10] X. Huang, Y. Wang, and L. Zhu, "One and three limit cycles in a cubic predator-prey system," Mathematical Methods in the Applied Sciences, vol. 30, no. 5, pp. 501-511, 2007.

[11] H. Cao and S. Fu, "Global existence and convergence of solutions to a cross-diffusion cubic predator-prey system with stage structure for the prey," Boundary Value Problems, vol. 2010, Article ID 285961, 24 pages, 2010.

[12] D. Henry, Geometric Theory of Semilinear Parabolic Equations, vol. 840 of Lecture Notes in Mathematics, Springer, Berlin, Germany, 1981.

[13] N. D. Alikakos, "An application of the invariance principle to reaction-diffusion equations," Journal of Differential Equations, vol. 33, no. 2, pp. 201-225, 1979.

[14] O. A. Ladyženskaja, V. A. Solonnikov, and N. N. Uralceva, Linear and Quasilinear Equations of Parabolic Type, vol. 23 of Translations of Mathematical Monographs, American Mathematical Society, Providence, RI, USA, 1968. 


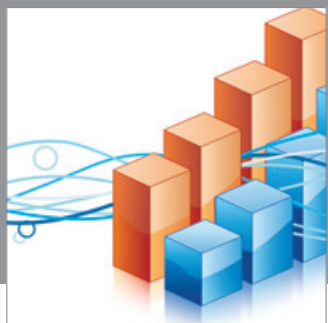

Advances in

Operations Research

mansans

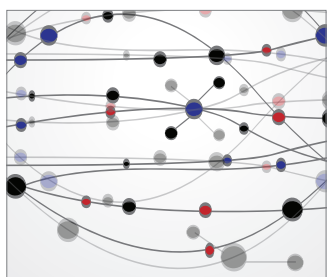

The Scientific World Journal
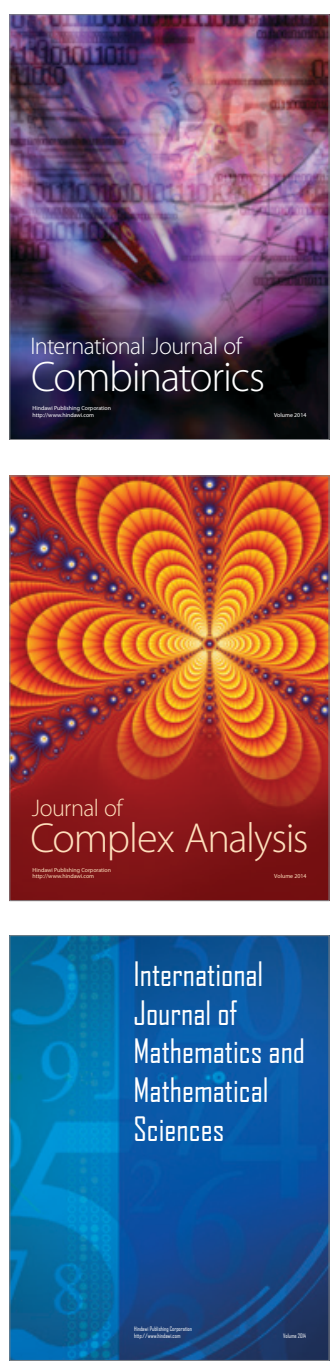
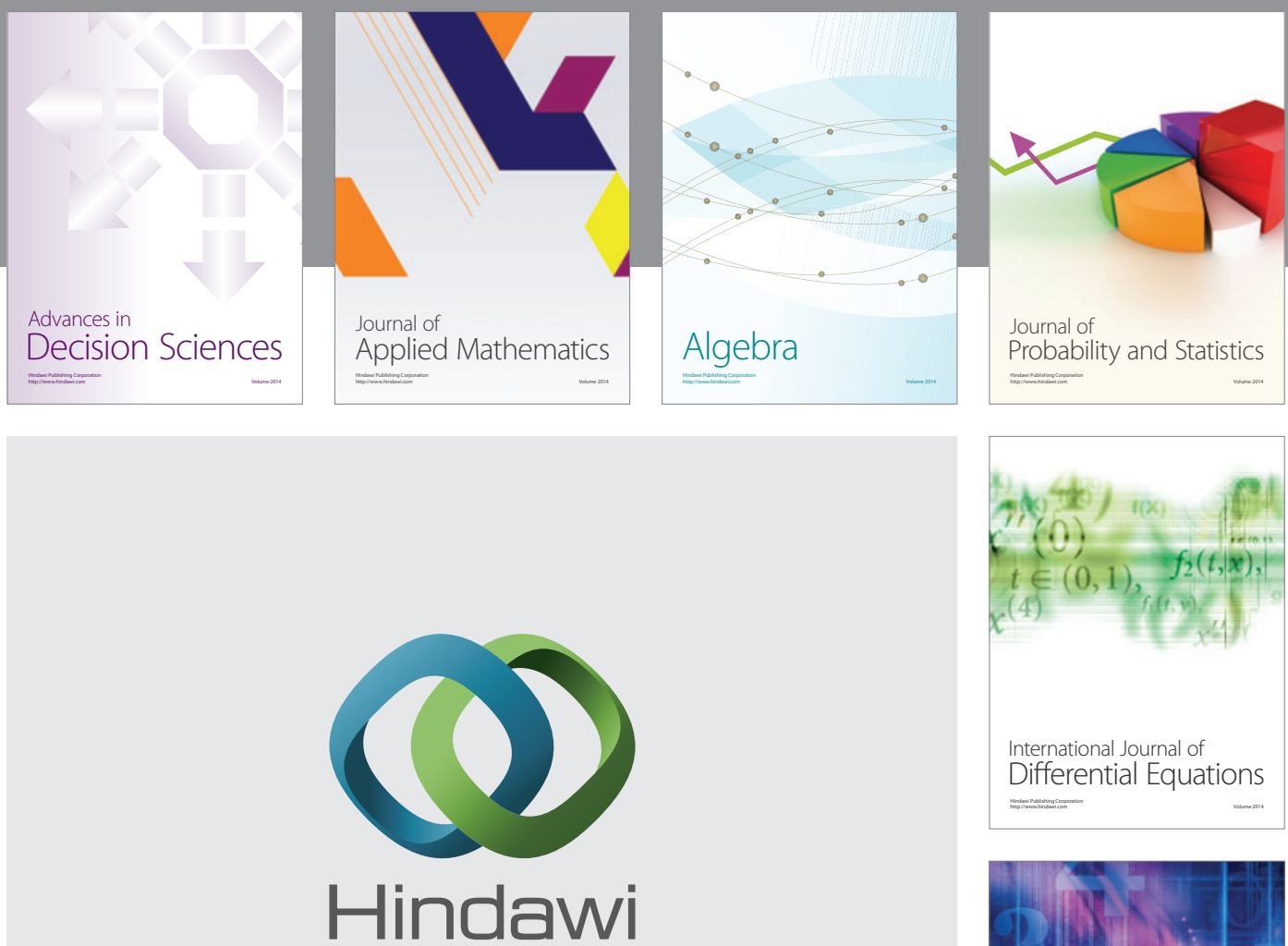

Submit your manuscripts at http://www.hindawi.com
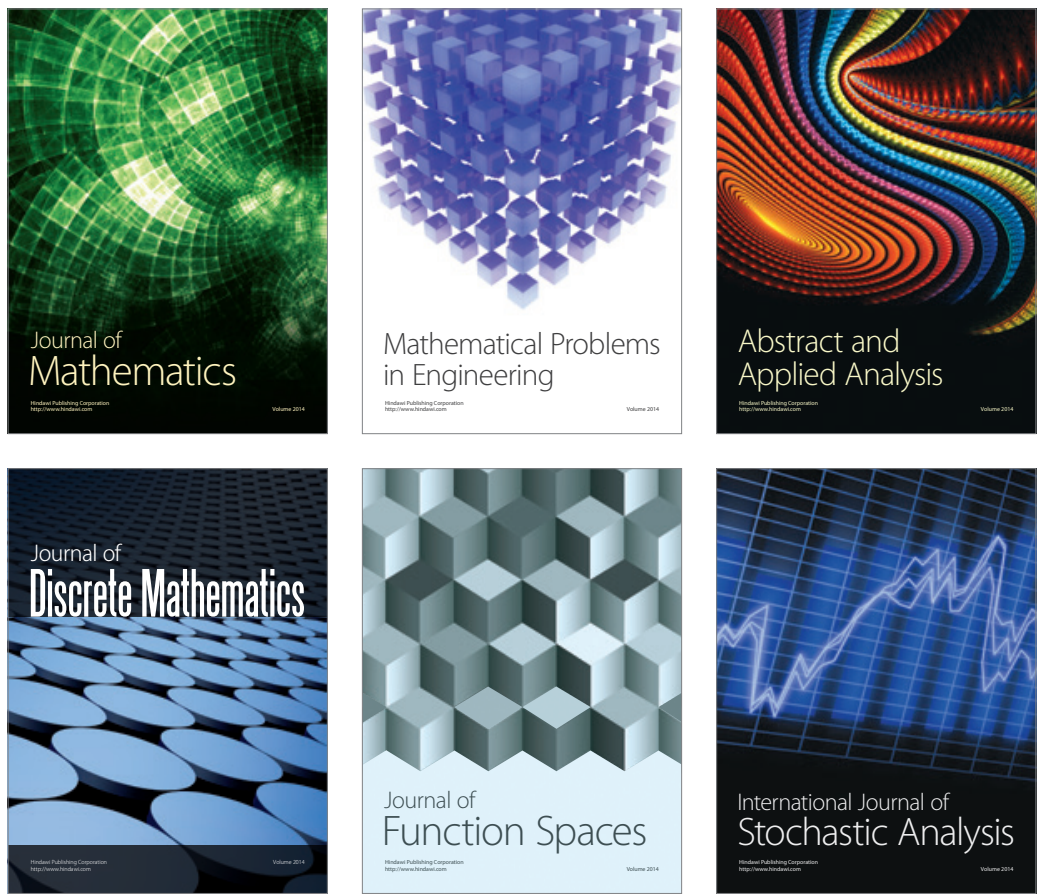

Journal of

Function Spaces

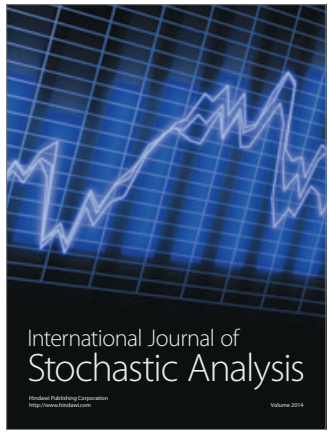

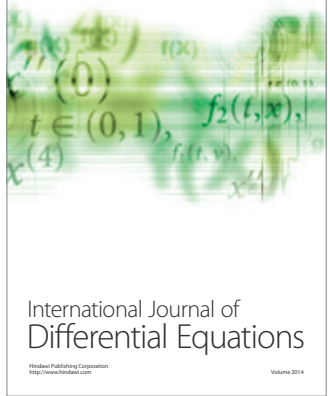
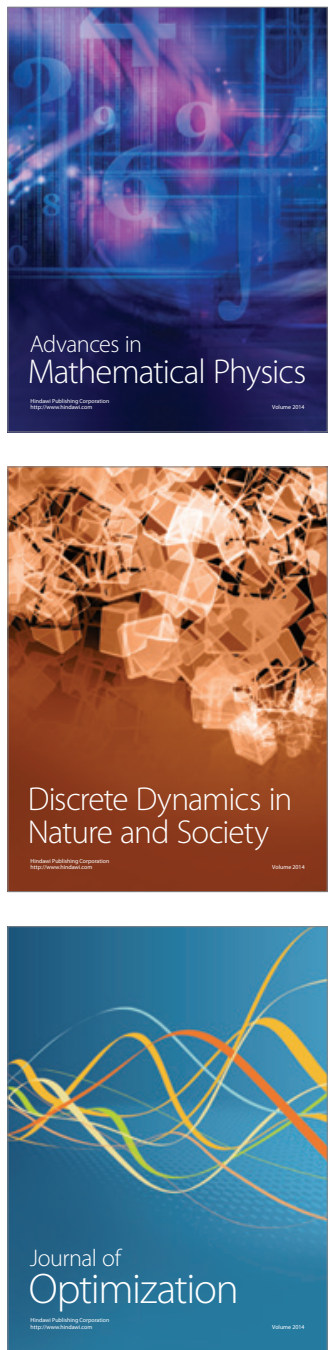